\title{
Pseudomyogenic hemangioendothelioma of bone treated with denosumab: a case report
}

\author{
Shinya Otani ${ }^{1}$, Robert Nakayama ${ }^{1 *}$ D, Tetsuya Sekita ${ }^{1}$, Toru Hirozane $^{1}$, Naofumi Asano ${ }^{1}$, Kazumasa Nishimoto $^{1}$, \\ Aya Sasaki ${ }^{2}$, Hajime Okita ${ }^{3}$, Hideo Morioka', Masaya Nakamura ${ }^{1}$ and Morio Matsumoto ${ }^{1}$
}

\begin{abstract}
Background: Pseudomyogenic hemangioendothelioma (PMHE) is a rare endothelial neoplasm that involves the bones in only $14 \%$ of all cases. The optimal treatment strategy has not been established. We herein report a case of primary PMHE in which denosumab treatment showed activity in both imaging studies and the clinical outcome.

Case presentation: A 20-year-old woman presented with worsening pain in her left ankle. Imaging studies showed multifocal fluorodeoxyglucose (FDG)-avid [maximum standardized uptake value (SUVmax), 15.95] osteolytic lesions in the bones of her left lower extremity. While waiting for the definitive pathologic diagnosis of PMHE, denosumab, a human immunoglobulin G2 monoclonal antibody against RANKL, was initiated to treat progressive bone absorption after curettage of one of the lesions. Denosumab induced osteosclerosis around the lesions and pain relief and was discontinued 4 years after its initiation. Although all of the multifocal lesions remained, they all became less FDG-avid (SUVmax, 2.6), and the patient developed no signs of new lesions or distant metastasis.
\end{abstract}

Conclusion: Denosumab plays a certain role in prevention of bone destruction by PMHE through suppression of osteoclast-like giant cells and would be an excellent treatment for bone absorption by PMHE of bone.

Keywords: Pseudomyogenic hemangioendothelioma, Denosumab, Osteoclast-like giant cells, FOSB

\section{Background}

Pseudomyogenic hemangioendothelioma (PMHE) is a rarely metastasizing endothelial neoplasm that most commonly occurs in young adults and often presents as multiple discontiguous nodules in different planes of a limb. This extremely rare vascular tumor was newly classified in the World Health Organization (WHO) classification of Tumors of Soft Tissue and Bone in $2013^{1) 2}$. The histological diagnosis of PMHE is often challenging, the optimal treatment has not been established, and the long-term prognosis is unclear. We herein report a case of 20-year-old woman with multiple intraosseous PMHE lesions in her left lower extremity that showed rapidly progressive osteolytic change. Although the multiple lesions did not disappear, denosumab administration led

\footnotetext{
*Correspondence: robert.a2@keio.jp

${ }^{1}$ Department of Orthopaedic Surgery, Keio University School of Medicine, 35

Shinanomachi, Shinjyuku, Tokyo 160-8582, Japan

Full list of author information is available at the end of the article
}

to pain relief, attenuation of the tumor viability, and formation of clear bone sclerosis around the lesions.

\section{Case presentation}

A 20-year-old woman with no medical history was referred to our hospital for evaluation and treatment of a 3 -month history of worsening pain in the left ankle. Physical examination revealed no remarkable findings except tenderness on the anterior aspect of her left ankle. All laboratory data were within the reference ranges. Radiographs showed multiple discontiguous osteolytic lesions in the bones of the left lower extremity (femur, patella, tibia, and talus) (Fig. 1a, b). The spotty lytic lesions were ill-circumscribed and lacked marginal sclerosis. Some were accompanied by thinning and ballooning of the bone cortexes (Fig. 1a, b). The lesion at the distal end of the tibia extended beyond the epiphyseal plate and eroded the subchondral bone; this lesion was considered to be the cause of her ankle pain. On ankle magnetic

(c) The Author(s). 2019 Open Access This article is distributed under the terms of the Creative Commons Attribution 4.0 International License (http://creativecommons.org/licenses/by/4.0/), which permits unrestricted use, distribution, and 


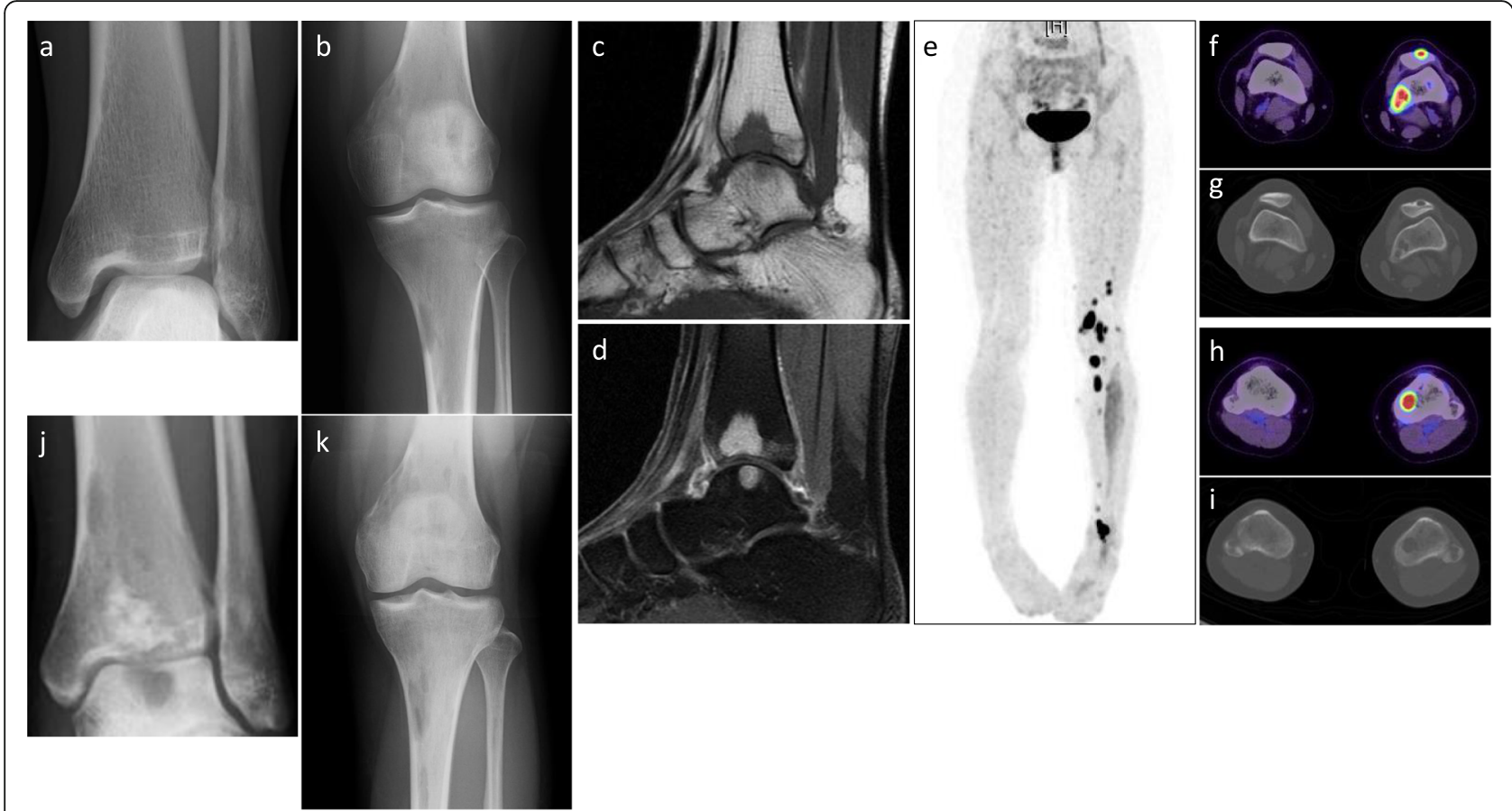

Fig. 1 Imaging findings at presentation. a and $\mathbf{b}$ Plain X-ray findings. Multifocal ill-circumscribed lytic lesions were seen in the left femur, patella, tibia, and talus. $\mathbf{c}$ and $\mathbf{d}$ Sagittal magnetic resonance imaging findings of the left ankle. The multifocal lesions showed $\mathbf{c}$ homogeneous low intensity on T1-weighted images and $\mathbf{d}$ homogeneous enhancement after gadolinium administration. e-i Fluorodeoxyglucose (FDG)-positron emission tomography/computed tomography showed multifocal FDG-avid lesions in the bones of the left lower extremity with a maximum standardized uptake value of 15.95. No lesions were found in the soft tissue. $\mathbf{j}$ and $\mathbf{k}$ Rapid progression after curettage and artificial bone grafting of the lesion at the distal end of the left tibia was observed. Each ill-circumscribed lytic lesion in the left extremity increased in size over 1 month

resonance imaging, the spotty lesions showed isointensity to the muscles on T1-weighted images (Fig. 1c), mild hyperintensity on T2-weighted images, and homogeneous enhancement by gadolinium administration (Fig. 1d). On fluorodeoxyglucose positron emission tomography (FDGPET), all of the intraosseous spotty lesions in the left lower extremity were FDG-avid with a maximum standardized uptake value (SUV) of 15.95; no such lesions were present in the soft tissues (Fig. 1e-i). Based on the findings from the imaging studies, the differential diagnoses of the multiple bone lesions included bone metastasis, hematological malignancies such as malignant lymphoma and multiple myeloma, and vascular tumors. Curettage of the lesion in the distal tibia and artificial bone grafting were performed for pain relief and histological diagnosis. While waiting for the definitive diagnosis, progressive bone absorption at all of the lesions and worsening lower limb pain occurred over 1 month (Fig. 1j, k). The preliminary pathology report suggested a non-hematological neoplasm composed of spindle cells and osteoclast-like giant cells, which led to a clinical diagnosis of bone metastases from a solid cancer despite the fact that no lesion suspicious of primary cancer was present. Based on the clinical diagnosis of bone metastasis, monthly administration of denosumab (120 mg) was initiated. The final pathological report demonstrated proliferation of spindle cells and epithelioid cells with eosinophilic cytoplasm. The tumor cells were immunohistochemically positive for keratin (AE1/AE3), CD31, ERG, and FOSB (Fig. 2a-d), which led to the diagnosis of PMHE. Because her limb pain gradually improved after denosumab administration, the monthly denosumab treatment was continued. After 1 year of denosumab treatment, the drug was administered at longer intervals of up to 6 months. The drug was discontinued 4 years after its initiation. Although the spotty lesions remained, a plain radiograph showed no increase in the size of the lesions or the surrounding marked marginal scleroses (Fig. 3a, b). On FDG-PET, the lesions showed clearly decreased SUVs (Fig. 3c-g). Because denosumab treatment led to dramatic symptom improvement, the patient did not agree to undergo a second-look biopsy or curettage of the remaining lesions at the time of denosumab discontinuation. The patient developed no signs of new lesions or distant metastasis and agreed to continue undergoing active surveillance.

\section{Discussion and conclusions}

PMHE is an extremely rare vascular tumor that was first reported by Mirra et al. [1] as a fibroma-like variant of epithelioid sarcoma in 1992. It was first defined by 

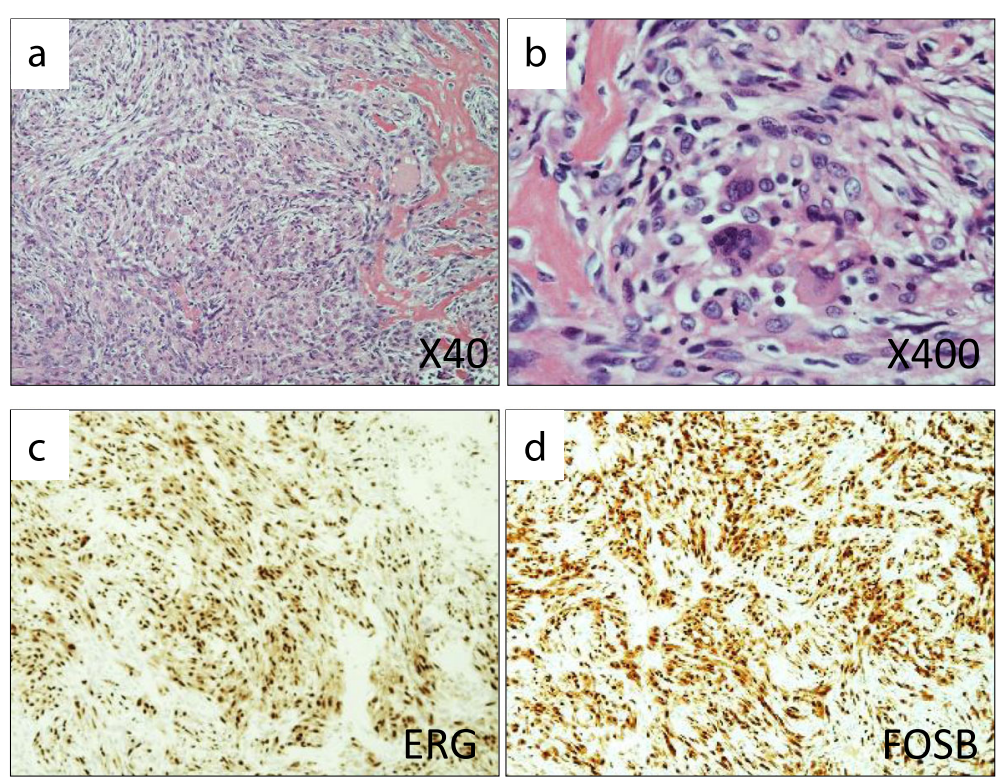

Fig. 2 Pathological findings. a and b Hematoxylin-eosin staining showed proliferation of spindle and epithelioid cells with eosinophilic cytoplasm, which was compatible with the diagnosis of pseudomyogenic hemangioendothelioma. $\mathbf{c}$ and $\mathbf{d}$ Immunohistochemical studies showed that the tumor cells were positive for ERG and FOSB

Hornick and Fletcher [2] in 2011 and newly classified in the 4th edition of the WHO classification of soft tissue tumors in 2013 [3]. Hornick and Fletcher [2] reported the largest case series involving 205 total lesions in 50 patients, which showed a striking male predominance (4.6:1.0) and a relatively young age at onset (mean, 31 years; $82 \%$ of patients were $\leq 40$ years old). Nearly half of the tumors arose in the lower extremities (54\%), and two-thirds (66\%) were multifocal lesions (range, 2-15 lesions). The lesions involved multiple tissue planes in $64 \%$ of cases, including the dermis, subcutis, muscle, and bone. Only $14 \%$ of the tumors were in the bones, and the tumor involved the bone alone in only one case [2]. Inyang et al. [4] reported a case series of 10 patients with

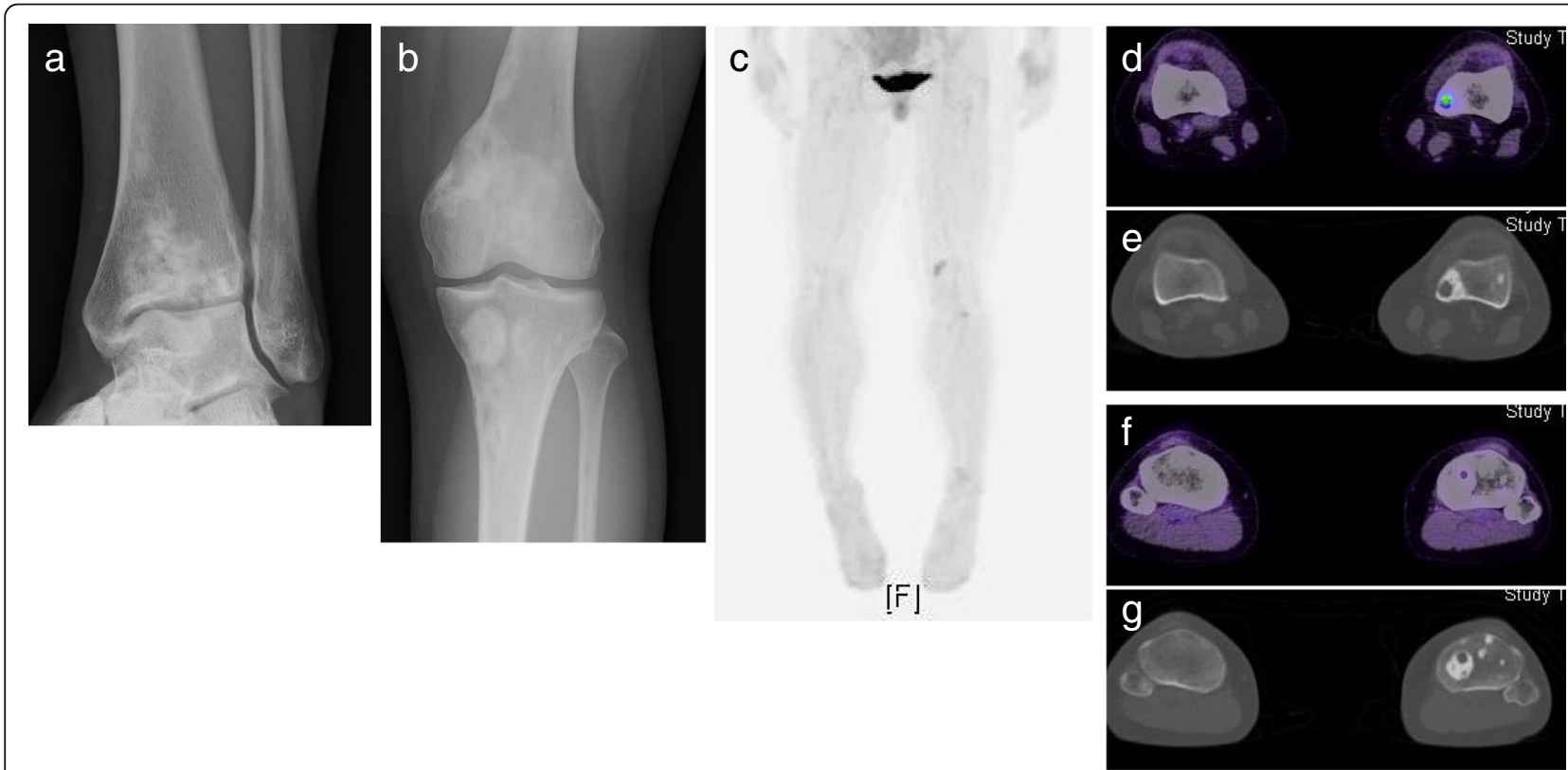

Fig. 3 Imaging findings 4 years following denosumab treatment. a and $\mathbf{b}$ Plain radiographs showed shrinkage of the lesions with striking marginal sclerosis. c-g Fluorodeoxyglucose (FDG)-positron emission tomography/computed tomography showed that each lesion had become less FDG-avid with a maximum standardized uptake value of 2.6 and was accompanied by remarkable sclerotic bone 
primary PMHE of bone and found patient demographics similar to those described by Hornick and Fletcher [2]. Plain radiographs showed multiple discontinuous osteolytic and circumscribed lesions, and pathological findings showed that $60 \%$ of the tumors had osteoclastlike giant cells [4]. Although the lesions were in one lower extremity, where PMHE commonly occurs, the present case was exceptional in terms of the sex of the patient (female) and the tissue plane involved (bone alone). Twenty cases of primary bone PMHE, including the present case, have been reported in the English-language literature (Table 1) [2, 4-12]. Most of the tumors were initially thought to be multiple bone metastases or multiple myeloma and were subsequently diagnosed as PMHE. Solitary lesions were seen in only three cases (15.0\%). Multiple lesions in a lower extremity were seen in half of the cases (10/20). No definitive evidence distinguished multiple bone metastases of PMHE from the multifocal discontiguous primary lesions that are often seen in vascular tumors.

The extreme rarity of this vascular tumor and its less characteristic morphological features make the histological diagnosis of PMHE enormously challenging. PMHE is known to be immunohistochemically positive for keratin AE1/AE3 and the endothelial transcription factor ERG. The balanced translocation $\mathrm{t}(7 ; 19)(\mathrm{q} 22 ; \mathrm{q} 13)$, resulting in a SERPINE1-FOSB fusion, was recently identified in all cases of PMHE [13, 14]. This consistent genetic alteration is considered to be the cause as well as a useful diagnostic marker of PMHE, and the positivity of FOSB by immunohistochemistry is an excellent surrogate marker for the presence of the genetic alteration $[15,16]$. In the present case, the immunohistochemical positivity for FOSB in addition to CD31 and ERG finally led to the diagnosis of PMHE.

The optimal treatment for PMHE has not been established to date, and the long-term oncological outcome remains unclear. A wide variety of treatments have been conducted, including surgery, chemotherapy, and the combination of both. In terms of surgery, Hornick and Fletcher [2] reported that all tumors had infiltrative margins and that $58 \%$ of patients developed local recurrence or additional nodules in the same region after local excision. Despite the generally indolent nature of PMHE, amputations were required for local control in some cases $[2,4]$, and systemic progression was seen in other cases. Conversely, two cases of spontaneous regression have been reported $[4,7]$. In terms of chemotherapy, the efficacy of gemcitabine and docetaxel and everolimus $[10,12]$ has been reported.

Denosumab is a human immunoglobulin G2 monoclonal antibody specific to receptor activator of nuclear factor kappa-B ligand (RANKL) and has been used for the treatment of osteoporosis, bone metastases, and giant

Table 1 Cases of primary bone pseudomyogenic hemangioendothelioma

\begin{tabular}{|c|c|c|c|c|c|c|c|}
\hline Case & Author & Year & Age & Sex & Location & Laterality & Comments \\
\hline 1 & Hornick & 2011 & 35 & Male & Finger & Solitary & \\
\hline 2 & Sheng & 2012 & 10 & Female & Lower extremety, multiple & One-sided & \\
\hline 3 & McGinity & 2013 & 25 & Male & T4 spine & Solitary & \\
\hline 4 & Bryanton & 2015 & 59 & Male & Multiple & Both & \\
\hline 5 & Righi & 2015 & 66 & Female & Lower extremety, multiple & One-sided & Curettage \\
\hline 6 & Shah & 2015 & 86 & Male & Lower extremety, multiple & One-sided & Pulmonary mets \\
\hline 7 & Joseph & 2015 & 45 & Male & Illum & Solitary & \\
\hline 8 & Inyang-1 & 2016 & 59 & Male & Multiple & Both & Complete metabolic resolution \\
\hline 9 & Inyang-2 & 2016 & 19 & Male & Lower extremety, multiple & One-sided & \\
\hline 10 & Inyang-3 & 2016 & 47 & Male & Lower extremety, multiple & One-sided & \\
\hline 11 & Inyang-4 & 2016 & 14 & Male & Upper extremety, multiple & One-sided & \\
\hline 12 & Inyang-5 & 2016 & 74 & Male & Trunk, multiple & One-sided & Spinal metastasis \\
\hline 13 & Inyang-6 & 2016 & 20 & Male & Femur, multiple & One-sided & \\
\hline 14 & Inyang-7 & 2016 & 66 & Male & Trunk, multiple & Both & \\
\hline 15 & Inyang-8 & 2016 & 12 & Male & Lower extremety, multiple & One-sided & \\
\hline 16 & Inyang-9 & 2016 & 26 & Male & Multiple & Both & Brain metastasis \\
\hline 17 & Inyang-10 & 2016 & 5 & Female & Hip and pelvis, multiple & One-sided & \\
\hline 18 & Krebs & 2017 & 33 & Male & Lower extremety, multiple & One-sided & \\
\hline 19 & Ozeki & 2017 & 15 & Male & Lower extremety, multiple & One-sided & Treated with everolimus. Spinal metastases \\
\hline 20 & Present & & 20 & Female & Lower extremety, multiple & One-sided & \\
\hline
\end{tabular}


cell tumors of bone [17-19]. In this case, all lesions showed simultaneous rapid progression of bone destruction that resulted in exacerbation of the limb pain after curettage. Denosumab was administered under the clinical diagnosis of bone metastasis to prevent further bone destruction. Given the indolent nature of the disease after diagnosis and the fact that most patients in the literature presented with pain associated with bone destruction, it is likely that each PMHE tumor of bone is induced to progress at some point by unknown triggers in its natural course. Reactive osteoclast-like giant cells have been seen in more than $60 \%$ cases [4], which are thought to be activated by FOSB-positive tumor cells by the unknown mechanism and considered responsible for the lytic destruction of the bone. Although denosumab did not show an essential anti-tumor effect because all of the lesions remained, we believe that denosumab played a certain role in suppression of the bone absorption by osteoclast-like giant cells and subsequent pain relief. The formation of highly characteristic osteosclerosis around the lesions could be the corroborating evidence. The precise mechanism of denosumab's activity in PMHE of bone was not clear since the tumor was not evaluated histologically after denosumab treatment.

Despite strikingly decreased SUVs, FDG-PET still showed multiple discontiguous nodules 4 years after initiation of denosumab treatment. The stable oncologic course of the case could be attributed to the indolent nature of the tumor. Deciding the optimal duration of denosumab treatment in such a young female patient with possibly indolent disease was enormously difficult. Denosumab was gradually withdrawn considering the substantial risk of osteonecrosis of the jaw due to the years-long denosumab administration, and also considering the teratogenic potency of the drug for future pregnancy. The patient developed no signs of new lesions, distant metastasis or progression of the bone absorption even after the termination of denosumab. Close long-term follow-up is still necessary, and denosumab treatment will be considered if the bone absorption recur.

\section{Abbreviations \\ FDG: Fluorodeoxyglucose; PET: Positron emission tomography; PMHE: Pseudomyogenic hemangioendothelioma; RANKL: Receptor activator of nuclear factor kappa-B ligand; SUVmax: Maximum standardized uptake value; WHO: World Health Organization}

\section{Acknowledgments}

We thank Prof. Tadashi Hasegawa for providing his expertise in pathological diagnosis, Angela Morben, DVM, ELS, from Edanz Group (www.edanzediting. $\mathrm{com} / \mathrm{ac}$ ), for editing a draft of this manuscript, and Eriko Hikawa for administrative support.

\section{Authors' contributions}

SO performed the data analysis and initial draft of the manuscript. AS and $\mathrm{HO}$ were responsible for histological review. SO, TH, NA, TS, KN, HM, MN, and MM performed the collection and assembly of data. SO and RN contributed to writing of the manuscript. RN and MM provided final approval of the manuscript. All authors read and approved the final manuscript.

Funding

No funding was received.

\section{Availability of data and materials}

All relevant data are provided in the manuscript.

\section{Ethics approval and consent to participate}

This study was approved by the ethics committee of Keio University School of Medicine. The patient provided signed informed consent for an Institutional Review Board-approved protocol for research use of medical records, of pathologic specimens obtained as part of routine clinical care, and publication.

\section{Consent for publication}

Written informed consent to publish the clinical data was obtained from the patient before the initiation of the study.

\section{Competing interests}

The authors declare that they have no competing interests.

\section{Author details}

${ }^{1}$ Department of Orthopaedic Surgery, Keio University School of Medicine, 35 Shinanomachi, Shinjyuku, Tokyo 160-8582, Japan. ${ }^{2}$ Department of Diagnostic Pathology, Keio University School of Medicine, Tokyo, Japan. ${ }^{3}$ Department of Pathology, Keio University School of Medicine, Tokyo, Japan.

Received: 18 April 2019 Accepted: 22 August 2019

Published online: 03 September 2019

\section{References}

1. Mirra JM, Kessler S, Bhuta S, Eckardt J. The fibroma-like variant of epithelioid sarcoma. A fibrohistiocytic/myoid cell lesion often confused with benign and malignant spindle cell tumors. Cancer. 1992;69(6):1382-95.

2. Hornick JL, Fletcher CD. Pseudomyogenic hemangioendothelioma: a distinctive, often multicentric tumor with indolent behavior. Am J Surg Pathol. 2011;35(2):190-201.

3. Fletcher CDM, World Health Organization, International Agency for Research on Cancer. WHO classification of tumours of soft tissue and bone. 4th ed. Lyon: IARC Press; 2013.

4. Inyang A, Mertens F, Puls F, Sumathi V, Inwards C, Folpe A, et al. Primary pseudomyogenic hemangioendothelioma of bone. Am J Surg Pathol. 2016; 40(5):587-98.

5. Sheng WQ, Wang J. Primary pseudomyogenic haemangioendothelioma of bone. Histopathology. 2012;61(6):1219-24.

6. McGinity M, Bartanusz V, Dengler B, Birnbaum L, Henry J. Pseudomyogenic hemangioendothelioma (epithelioid sarcoma-like hemangioendothelioma, fibroma-like variant of epithelioid sarcoma) of the thoracic spine. Eur Spine J. 2013;22(Suppl 3):S506-11.

7. Bryanton M, Makis W. Pseudomyogenic hemangioendothelioma mimicking multiple myeloma on 18F-FDG PET/CT, followed by spontaneous regression. Clin Nucl Med. 2015;40(7):579-81.

8. Righi A, Gambarotti M, Picci P, Dei Tos AP, Vanel D. Primary pseudomyogenic haemangioendothelioma of bone: report of two cases. Skelet Radiol. 2015;44(5):727-31.

9. Shah AR, Fernando M, Musson R, Kotnis N. An aggressive case of pseudomyogenic haemangioendothelioma of bone with pathological fracture and rapidly progressive pulmonary metastatic disease: case report and review of the literature. Skelet Radiol. 2015;44(9):1381-6.

10. Joseph J, Wang WL, Patnana M, Ramesh N, Benjamin R, Patel S, et al, Cytotoxic and targeted therapy for treatment of pseudomyogenic hemangioendothelioma. Clin Sarcoma Res. 2015:5:22.

11. Krebs S, Monti S, Lewis N, Luciano A, Rastrelli L, Mannelli L. Lower-extremity pseudomyogenic hemangioendothelioma on bone scintigraphy and PET/ CT. Clin Nucl Med. 2017:42(5):383-5.

12. Ozeki M, Nozawa A, Kanda K, Hori T, Nagano A, Shimada A, et al. Everolimus for treatment of pseudomyogenic hemangioendothelioma. J Pediatr Hematol Oncol. 2017;39(6):e328-31.

13. Walther C, Tayebwa J, Lilljebjorn H, Magnusson L, Nilsson J, von Steyern FV, et al. A novel SERPINE1-FOSB fusion gene results in transcriptional up- 
regulation of FOSB in pseudomyogenic haemangioendothelioma. J Pathol. 2014;232(5):534-40.

14. Trombetta D, Magnusson L, von Steyern FV, Hornick JL, Fletcher CD, Mertens F. Translocation t(7;19)(q22;q13)-a recurrent chromosome aberration in pseudomyogenic hemangioendothelioma? Cancer Genet. 2011;204(4):211-5.

15. Hung YP, Fletcher CD, Hornick JL. FOSB is a useful diagnostic marker for pseudomyogenic hemangioendothelioma. Am J Surg Pathol. 2017;41(5): 596-606.

16. Sugita S, Hirano H, Kikuchi N, Kubo T, Asanuma H, Aoyama T, et al. Diagnostic utility of FOSB immunohistochemistry in pseudomyogenic hemangioendothelioma and its histological mimics. Diagn Pathol. 2016; 11(1):75.

17. McClung MR, Lewiecki EM, Cohen SB, Bolognese MA, Woodson GC, Moffett $\mathrm{AH}$, et al. Denosumab in postmenopausal women with low bone mineral density. N Engl J Med. 2006;354(8):821-31.

18. Gul G, Sendur MA, Aksoy S, Sever AR, Altundag K. A comprehensive review of denosumab for bone metastasis in patients with solid tumors. Curr Med Res Opin. 2016;32(1):133-45.

19. Thomas D, Henshaw R, Skubitz K, Chawla S, Staddon A, Blay JY, et al. Denosumab in patients with giant-cell tumour of bone: an open-label, phase 2 study. Lancet Oncol. 2010;11(3):275-80.

\section{Publisher's Note}

Springer Nature remains neutral with regard to jurisdictional claims in published maps and institutional affiliations.

Ready to submit your research? Choose BMC and benefit from:

- fast, convenient online submission

- thorough peer review by experienced researchers in your field

- rapid publication on acceptance

- support for research data, including large and complex data types

- gold Open Access which fosters wider collaboration and increased citations

- maximum visibility for your research: over $100 \mathrm{M}$ website views per year

At $\mathrm{BMC}$, research is always in progress.

Learn more biomedcentral.com/submissions 